\title{
Osteochondritis dissecans of the elbow
}

\author{
Ryan W. Churchill ${ }^{1} \cdot$ Julianne Munoz $^{2} \cdot$ Christopher S. Ahmad ${ }^{2}$
}

Published online: 28 April 2016

(C) Springer Science+Business Media New York 2016

\begin{abstract}
Capitellar osteochondritis dissecans (OCD) can be a significant problem in adolescent overhead athletes. The cause is likely multifactorial secondary to repetitive stresses, biomechanical mismatch, and a tenuous vascular supply of the capitellum. Recent literature reveals that the prevalence is likely higher than previously thought. This, in conjunction with increased levels of athletic competition in children at younger ages, has fed the recent interest in this topic. The literature continues to show that non-operative treatment is still successful for stable lesions. Unstable lesions, therefore, have been the focus of the new literature regarding operative management and outcomes. The aim of this paper is to provide a summary of current literature and an up-to-date approach to the diagnosis, evaluation, and treatment of osteochondritis dissecans of the capitellum.
\end{abstract}

Keywords Osteochondritis dissecans · Capitellum · Osteochondral autograft transplantation system · Elbow arthroscopy $\cdot$ Microfracture $\cdot$ Overhead athletes

This article is part of the Topical Collection on Elbow Soft Tissue Surgery

Christopher S. Ahmad

csa4@cumc.columbia.edu

Ryan W. Churchill

ryan.w.churchill@gmail.com

Julianne Munoz

Julianne.marvin@gmail.com

1 Department of Orthopaedic Surgery, MedStar Georgetown University Hospital, 3800 Reservoir Road NW, Washington, DC 20007, USA

2 Department of Orthopaedic Surgery, Columbia University Medical Center, 622 West 168th Street, PH-1122, 10032 New York, NY, USA

\section{Introduction}

König coined the term osteochondritis dissecans (OCD) in 1888 when discussing a potential cause of loose bodies within a joint. He theorized three means by which loose bodies could be formed: direct trauma resulting in acute osteochondral fracture, osteonecrosis and fragmentation resulting from minimal trauma, or spontaneous development without evidence of trauma $[1,2]$. Today, OCD refers to an acquired lesion of the subchondral bone with varying degrees of resorption, fragmentation, and sclerosis with potential involvement of the overlying cartilage but not the result of an acute osteochondral fracture [2]. The etiology of these lesions is likely multifactorial with potential causes including repetitive trauma, vascular, inflammatory, and genetic factors [2]. The elbow, specifically the capitellum, is one of the most common sites of these lesions and the area where their incidence is increasing most rapidly $[3,4]$. Many treatment strategies exist for OCD in the elbow, including rest/activity modification, arthroscopic removal of loose bodies, abrasion chondroplasty, microfracture, retrograde drilling, fixation of fragments, osteochondral autograft transplantation system, and costal osteochondral autograft [5-7]. The aim of this paper is to evaluate and summarize recent advances in the literature in the diagnosis and treatment of OCD of the elbow and to serve as a guide for management.

\section{Background}

OCD of the elbow primarily affects the capitellum in adolescent athletes engaging in repetitive overhead activities and

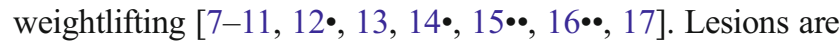
also rarely seen in the radial head, olecranon, and trochlea. A recent case report of trochlear OCD by Miyake et al. noted 
only 22 reported cases as of 2013 in the English literature [7, 18]. Therefore, this paper will focus on the recent literature regarding capitellar OCD.

OCD must be distinguished from Panner's disease, which also involves the capitellum. Panner's disease is a self-limiting condition of osteochondrosis of the capitellum. It primarily affects boys under 10 years old without history of trauma [6-8]. Patients present with activity-related pain and are tender along the lateral aspect of the elbow and the capitellum. Radiographs can show fissuring, lucencies, fragmentation, and change in the contour of the capitellum. Later imaging shows reossification which usually coincides with resolution of symptoms [6, 19]. The treatment of this disease is activity modification, rest, anti-inflammatory medications, and for severe symptoms, a brief period of immobilization with anticipated resolution of symptoms in most patients by 6-8 weeks $[6,19]$. Gradual return to activity may commence upon resolution of symptoms [6, 19].

The OCD patient population is slightly older than that of Panner's, and the condition is not self-limiting. Patients with capitellar OCD are usually between 11 and 17 years old and participate in repetitive overhead activities such as baseball, gymnastics, football, javelin, or overhead weightlifting [3, 7, $9,15 \bullet \bullet, 16 \bullet \bullet, 17,19,20 \bullet, 21-25]$. The radiocapitellar joint provides axial stability to the elbow with the capitellum transmitting approximately $60 \%$ of compressive forces across it [9, 22]. Overhead throwing and axial loading activities, such as gymnastics or weightlifting, generate repetitive radiocapitellar compressive forces [7, 9, 19, 22]. In overhead throwers, repetitive shear forces are also transmitted across the radiocapitellar joint, primarily during the late cocking and early acceleration pitching phases $[7,9,19,22]$. In the largest study to date, Kida et al. investigated the prevalence and characteristics of capitellar OCD lesions in 2433 adolescent baseball players between the ages of 12 and 18. They found that adolescent baseball players who started competition at a younger age and played for a longer time were at risk for developing capitellar OCD lesions. Furthermore, the prevalence of OCD was $3.4 \%$, which was significantly higher than the $1.3-1.6 \%$ prevalence from previous literature [16••].

The anatomy of the radiocapitellar joint and blood supply to the capitellum are thought to play a significant role in the development of these lesions. Schenck et al. performed a cadaveric study evaluating the biomechanical characteristics of the radial head and the capitellum. Stiffness of the capitellar articular surface decreased from medial to lateral. Furthermore, the central portion of the radial head was significantly stiffer than the lateral capitellum creating a biomechanical mismatch that could lead to increased strain on the lateral aspect of the capitellum during loading activities [26]. Haraldsson et al. studied the vascular anatomy of the distal humerus and its relationship to OCD development, noting that the capitellar blood supply entered via the posterior aspect of the distal humerus by way of two arteries, radial recurrent branches, and interosseous recurrent branches [7, 19, 27]. This aspect of the vascular anatomy along with an immature capitellum lacking substantial metaphyseal collateral flow creates a potential situation where blood flow can be disrupted in the setting of repetitive trauma [7, 19]. Thus, the development of OCD is multifactorial: a combination of repetitive stresses applied across the radiocapitellar joint, the biomechanical mismatch of the radial head and capitellum, as well as a tenuous vascular supply.

\section{Evaluation}

The patient with capitellar OCD usually presents with progressive worsening of activity-related pain and stiffness [7, $16 \bullet \bullet, 19]$. In the overhead throwing athlete, the dominant throwing arm is affected. Kida et al. noted that $32.9 \%$ of adolescent baseball players reported elbow pain with throwing at the time of the study and $81.7 \%$ reported prior instances of elbow pain but had played through having felt it was not severe enough. These patients who played through pain presented with higher grade osteochondral lesions [16••]. Occasionally, patients report mechanical symptoms, such as catching, clicking, and locking, which are concerning for loose bodies within the joint. On physical exam, patients most often have tenderness over the radiocapitellar joint and may lack $15-30^{\circ}$ of extension $[6,7,19]$. Patients may have crepitus, especially with pronation and supination. Similarly, a positive radiocapitellar compression test may occur, where active pronation and supination with the elbow in extension reproduces pain at the radiocapitellar joint $[6,7,19]$.

Initial imaging for OCD evaluation includes three-view plain films of the elbow: extension anterior-posterior (AP), $45^{\circ}$-flexion AP, and lateral. Initially, X-rays may be negative but later capitellar findings include lucencies, flattening, sclerosis, and fragmentation along with intra-articular loose bodies. These changes are usually seen in the anterolateral aspect of the capitellum [7, 19]. Several classification systems exist for osteochondral lesions based on plain radiographs. Claessen et al. evaluated the interobserver reliability of a sample of these classifications. They provided orthopaedic surgeons and musculoskeletal radiologists with imaging studies including standard X-rays and computerized tomography (CT) scans of 22 cases of capitellar OCD and requested lesion classification according to four schemes: Minami, Berndt and Harty, Ferkel and Sgaglione, and Anderson. The Minami classification was the most reliable but still only had fair interobserver agreement. None of the classifications fared well when applied without magnetic resonance imaging (MRI) evaluation [12•]. Prior to this, Kijowski and De Smeet reported poor sensitivity for diagnosis or characterization using plain 
radiographs alone. Only $66 \%$ of their patients with known capitellar OCD had successful diagnosis on the initial Xrays [28].

MRI is the best imaging modality for evaluating OCD lesions and can demonstrate early-stage lesions when radiographs may appear normal [7, 19, 29]. The earliest MRI findings are uniform low-signal-intensity changes in the superficial capitellum on T1-weighted imaging with normal T2 imaging. As the lesion progresses, changes are seen on $\mathrm{T} 1$ and T2 imaging [7, 19, 29]. Gadolinium contrast enhancement of the OCD lesion suggests vascularity of the fragment and good viability [7]. The capitellar pseudodefect is a normal anatomic variant worth distinguishing on MRI. It occurs posterolaterally as opposed to the more anterolateral OCD lesion [7, 19, 29].

De Smet et al. were the first to report MRI characteristics of unstable OCD lesions, originally involving the knee [30]. Kijowski later modified these criteria for identifying unstable juvenile OCD lesions in the knee [29]. Kijowski and De Smet eventually applied these criteria to capitellar OCD and found that they correlated with surgical findings. Lesions that were unstable at surgery had a thin line of high-signal intensity between the OCD lesion and the underlying bone and a discrete round high-signal intensity area under the OCD lesion indicating a cyst on T2 imaging [31]. Jans et al. investigated the ability of the Kijowski criteria to evaluate the stability of capitellar OCD lesions in 25 patients. The presence of all criteria, namely high-T2-signal rim, surrounding cysts, a fluid-filled osteochondral defect, and a thin high-T2intensity fracture line was $100 \%$ sensitive for unstable lesions [32]. Iwasaki et al. retrospectively reviewed the MRIs of 27 overhead athletes who underwent surgery for capitellar OCD. De Smet's original criteria afforded $89 \%$ sensitivity for predicting unstable lesions, which they concluded, were inferior to surgical evaluation for stability [23]. Satake et al. also recently evaluated the ability of imaging studies, including MRI, to predict intraoperative stability of lesions and found that preoperative MRI directly related to the intraoperative stability of lesions [33]. As such, MRI should still be considered the best imaging tool available for evaluating OCD lesion stability.

Ultrasound has recently garnered attention as another diagnostic tool. Kida et al. studied the prevalence of OCD in adolescent baseball players using ultrasound examination as the initial imaging on all 2433 study participants. They utilized the method described by Harada et al. in 2006 for visualizing the capitellum. Long- and short-axis views along the anterior and posterior aspect of the capitellum were utilized to evaluate the articular surface and subchondral bone [16••, 34]. They reported $100 \%$ positive predictive value for their ultrasound screening and suggested that a distinctive loss of the smooth articular surface on ultrasound was a good indicator of an osteochondral lesion [16••]. While ultrasound provides a quick screening tool, it still remains a user-dependent modality that requires significant training for diagnostic acumen [35, 36].

\section{Treatment}

The initial approach to treatment of capitellar OCD is determined by the stability of the lesion. Numerous classification systems exist for this purpose utilizing physical exam, imaging, and intraoperative findings. Takahara et al. found that stable lesions occurred in patients who had an open capitellar physis, localized flattening or radiolucency, and good elbow range of motion at initial presentation. These patients responded well and healed with non-operative treatment whereas patients with unstable lesions (a closed capitellar physis, fragmentation on X-rays, and a range of motion lacking more than $20^{\circ}$ ) had better outcomes with operative intervention [37]. While Takahara and colleagues did not use MRI characteristics in their assessment of the stability of the OCD lesion, other authors have strongly advocated the use of the MRI characteristics described in the above sections to add to the stability assessment $[6,7,13,19,21,22,29,32,33,38$, 39]. Thus, generally, the stability of a lesion is determined by the status of the capitellar physis, the size and location of the lesion, and the nature of the lesion based on imaging [19].

\section{Non-operative treatment}

Patients with stable lesions, as previously stated, are initially treated non-operatively. This involves immediate cessation of aggravating activities that load the radiocapitellar joint, i.e., throwing, gymnastics, weightlifting [6, 7, 19, 40, 41]. Some authors advocate the use of a hinged elbow brace for a period of 1-6 weeks in the initial resting period to allow intermittent range of motion exercises to prevent stiffness, while others recommend rest without immobilization $[6,19]$. After an initial period of rest, physical therapy is initiated avoiding strengthening until resolution of symptoms [19]. Most athletes who respond to conservative management may start gentle overhead throwing at 3-4 months with return to play at 6 months $[6,7,19,41]$.

Matsuura et al. revealed that patients with early-stage, stable OCD lesions who were compliant with conservative therapy had an $84.2 \%$ healing rate while those who had similar lesions but were noncompliant with conservative therapy had a $22.7 \%$ healing rate. Those patients who did not improve with 6 months of conservative therapy went on to surgery [40]. Griewe et al. reported that patients with not only abnormal T1 signals but also abnormal T2 signals on MRI without rim enhancement or obvious defect (both indicative of stable lesions) can initially be treated conservatively. They 
advocated for a follow-up MRI in patients with abnormal T1 and T2 signals to reassess the lesion at 2-3 months into conservative management. If there was no improvement after 36 months of conservative treatment, then surgery should be considered [19].

\section{Operative treatment}

Surgery is indicated for patients who fail conservative management, have unstable lesions on exam and imaging, or have loose bodies with associated mechanical symptoms $[6,7,13$, 14., 19]. Potential surgical interventions include arthroscopic removal of loose bodies, abrasion chondroplasty, retrograde drilling, microfracture, in situ fixation, osteochondral autograft transplantation system (OATS), and costal osteochondral transplantation (COT) $[5-7,10,13,14 \cdot, 15 \bullet, 20 \bullet, 22,42]$. The most appropriate surgical intervention depends on many factors including lesion size, amount of lateral extension, and the presence of a stable cartilage cap [6-8, 19, 37].

\section{Arthroscopic loose body removal/abrasion chondroplasty}

Arthroscopic loose body removal and abrasion chondroplasty remain viable surgical options. This procedure entails debridement of the defect to a stable cartilage rim and removal of loose bodies within the joint. The results are variable. Takahara found that patients who underwent loose body removal without a reconstructive procedure when greater than $50 \%$ of the capitellar surface was involved had worse outcomes [37]. Previous studies have found inferior results when the lesion extends into the lateral capitellum and compromises the lateral column $[6-8,17,19,22,42]$. Tis et al. recently looked at outcomes of arthroscopic procedures including microfracture and retrograde drilling and included chondroplasty and loose body removal. Pain persisted in $33 \%$ of patients, and despite some returning to physical activity, many did not achieve previous levels [9]. Other studies have demonstrated reliable pain relief and improved range of motion but also report that patients may not be able to return to pre-injury levels of athletics [43-47]. This suggests different management for lesions greater than $50 \%$ of capitellar surface, those that extend into the lateral capitellum, and patients with high athletic expectations.

\section{Microfracture/retrograde drilling}

Microfracture and retrograde drilling remain good options for OCD lesions that do not extend into the lateral column. Retrograde drilling is used to treat unstable OCD lesions with stable cartilage caps. A 0.062-in. K-wire is drilled retrograde from the posterolateral, distal humerus into the OCD lesion under fluoroscopic guidance taking care not to violate the articular cartilage. Using the first $\mathrm{K}$-wire as a guide, the subchondral bone is drilled anywhere from one to four more times to stimulate a marrow healing response $[6,9]$. Microfracture can be used to treat lesions without lateral column extension even when overlying cartilage is disrupted. In this technique, after the lesion bed has been debrided to a stable rim, an awl, or K-wire (0.045- or 0.062-in.) is used to create holes within the lesion to stimulate marrow healing and a fibrocartilage response [6, 9, 13] (Fig. 1).

Wulf and colleagues reviewed MRIs 1 year out from microfracture to assess fibrocartilaginous healing response in correlation with patient outcomes. Mean OCD lesion size was $98 \mathrm{~mm}^{2}$. Fibrocartilage growth restoring articular congruity occurred in $80 \%$ of patients. Two patients did not return to prior levels of athletics. The average time to return to sport was 5.1 months with all patients showing improved functional outcome scores [39]. In Lewine et al., average OCD lesions were $9.8 \mathrm{~mm}$ in the coronal plane, $9.1 \mathrm{~mm}$ in the sagittal plane, and $3 \mathrm{~mm}$ in depth. Patients underwent drilling and microfracture, and all had improved range of motion and functional outcome scores. Return to any sport occurred in $85.7 \%$ of patients and return to primary sport in $66.7 \%$. This is consistent with previous return to sport data [48]. Bojanic and colleagues found improved functional outcomes with microfracture in teenage athletes. All but one patient had an excellent result based on the Mayo elbow performance score. Furthermore, greater than half of the patients returned to previous levels of sport or higher [49]. Microfracture and retrograde drilling remain good options with consistent, reproducible results in shallow OCD lesions without lateral column involvement.

\section{In situ fixation}

Several methods of in situ fixation of unstable OCD lesions exist. Fixation options include Herbert screw fixation, Kwires, bioabsorbable pins, and bone pegs [7, 14•, 21, 38]. This is a valuable technique because it avoids the need for an arthrotomy for fixation. In a review by Baker et al., union rates were as high as $82-100 \%$ and pre-injury level return to sport at $68-100 \%$ [7]. Hennrikus et al. retrospectively reviewed 26 cases of large, unstable in situ OCD lesions with mean width and thickness of 12.0 and $5.4 \mathrm{~mm}$, respectively. Twenty of 26 cases achieved union. In the six requiring revision, mean age ( 15.3 years) and sagittal lesion width (greater than $13 \mathrm{~mm}$ ) were significantly associated with nonunion. Two-thirds of patients returned to pre-injury level of sport, and all patients had improved outcome scores. In all but two 
Fig. 1 Arthroscopic images of patient with capitellar osteochondritis dissecans lesion with multiple loose bodies. Images demonstrate arthroscopic removal of loose bodies $(\mathbf{a}, \mathbf{b})$ and subsequent OCD lesion site after debridement to a stable edge (c). d The bleeding after microfracture indicating satisfactory penetration of subchondral bone
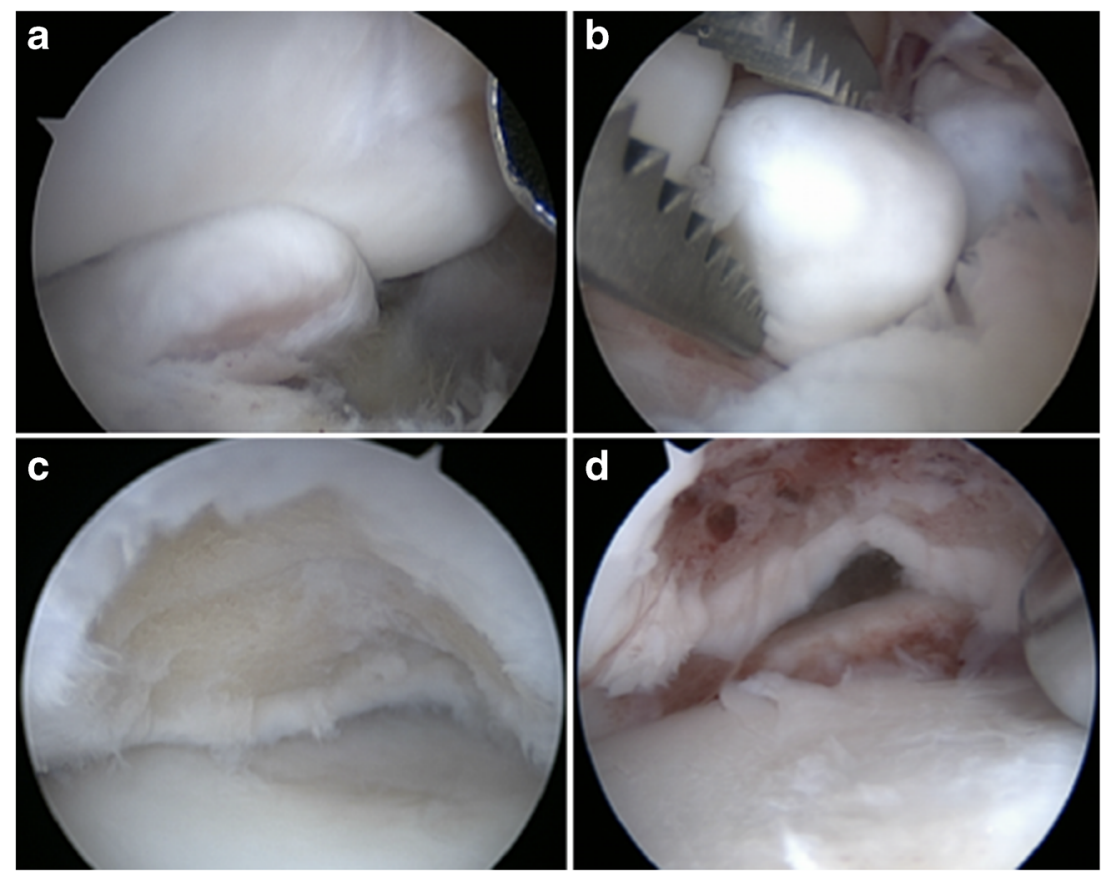

of the cases, bioabsorbable pins were used which lead to the authors' final endorsement of this form of fixation [21].

Uchida et al. used hydroxyapatite (HA)/poly-L-lactate acid (PLLA) threaded pins during arthroscopic in situ fixation of OCD lesions in 18 adolescent baseball players. Seventeen patients had excellent outcomes, and at 3-year follow-up, $94 \%$ had returned to sports, 15 of whom at the same level or higher. All lesions radiographically united. The authors suggest that lesions greater than $50 \%$ of the articular surface warrant consideration for osteochondral transplantation since at this size they predispose to osteoarthritis [14•]. While in situ fixation is a valuable treatment option for larger, unstable OCD lesions, when it is not technically feasible due to fragment size, osteochondral transplantation should be considered next.

\section{Osteochondral autograft transplantation system}

OATS has become a popular topic in recent OCD literature. Indications include large, unstable lesions, lesions involving greater than $50 \%$ of the articular surface, and lesions extending into the lateral border of the capitellum and involving the lateral column. Lateral condylar extension requires reconstructive techniques that provide subchondral support and prevent the radial head from engaging the lesion [6, 7, 19, 37]. OATS involves harvesting donor osteochondral plugs from non-weight-bearing portions of the knee, usually from the superior aspect of the lateral femoral condyle or trochlea, via a small arthrotomy. Schub et al. evaluated MRI mapping of articular cartilage thickness in the knee and elbow. The distal, anterolateral femoral condyle, and the posterior pole of the medial femoral condyle were the closest in cartilage thickness to the elbow although the cartilage layer at both sites was still thicker than any site in the elbow [11]. The recipient site in the capitellum is prepared by coring out the lesion usually to depth of $10 \mathrm{~mm}$ [10]. The donor plug(s) is/are then placed and impacted until flush with surrounding cartilage. This technique has the advantage of replacing the defect with hyaline cartilage $[8,10]$. The process is repeated until the OCD lesion is adequately restored (Fig. 2).

Early studies show return to pre-injury level of play after OATS occurs in 75-92.6\% patients [20•, 22]. Kosaka et al. studied 32 male athletes treated with bone peg fixation versus OATS for large capitellar OCDs. Range of motion and outcome scores improved with $81.3 \%$ of patients returning to prior levels within the first year after surgery. Bone peg fixation was more likely to fail and yielded worse results especially in the presence of significant lateral extension [22]. Similarly, Maruyama et al. evaluated 33 male baseball players treated with OATS for capitellar OCD. One to three osteochondral plugs were used with an average diameter of $7 \mathrm{~mm}$. All patients achieved graft incorporation by an average of 3.8 months, and all patients had improved outcome scores with $91 \%$ reporting no pain at follow-up. Thirty-one of the 33 patients returned to pre-injury level of play at an average of 6.9 months. The authors credit their sooner return to play figures because of larger graft size, which was nearly twice the diameter of those previously described [15••]. Lyons et al. examined OATS for capitellar OCD in competitive high 
Fig. 2 Arthroscopic images of capitellar osteochondritis dissecans lesion (black arrow) after debridement (a), preparation for osteochondral autograft plug (b), and subsequent placement of graft (c)
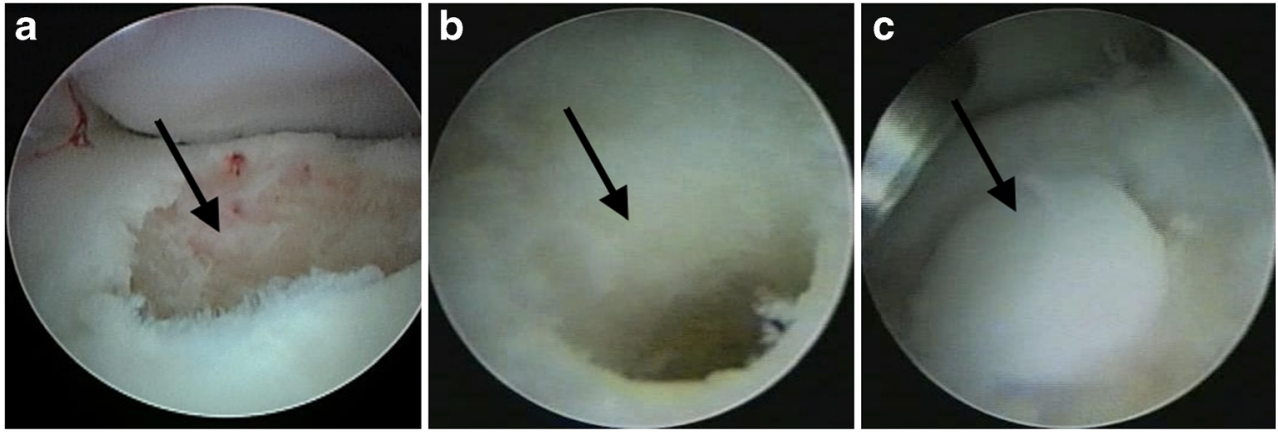

school athletes. All lesions were greater than $1 \mathrm{~cm}^{2}$. Outcome scores and range of motion improved in all patients with return to sport at an average of 4.4 months. These authors also utilized larger plugs, favoring one large plug of $10 \mathrm{~mm}$, if possible, over mosaicplasty for greater graft support perhaps contributing to quicker return to play [20 $0^{\bullet}$.

Donor site morbidity and dual arthrotomies of the elbow and knee are downsides to OATS. Several recent studies have shown little to no residual knee pain in the short or medium terms after OATS in athletes if donor grafts are kept at or below $10 \mathrm{~mm}$ in diameter [15••, 20•, 22, 50, 51•]. Gancarczyk et al. performed a cadaveric study attempting arthroscopic OATS for capitellar lesions to avoid arthrotomy. In central lesions, arthroscopic OATS was successful with an average perpendicularity of $85.3^{\circ}$ and for lateral lesions at $85.8^{\circ}$. They were successful in lesions as high as $75-80^{\circ}$ anterior to the humeral shaft. This is significant given that the majority of lesions occurs $45-60^{\circ}$ anterior to the humeral shaft. The ability to achieve greater than satisfactory perpendicularity arthroscopically throughout the arc that most of these lesions occur may help avoid arthrotomy [51•].

\section{Costal osteochondral transplantation}

COT is an innovative solution for lateral column OCD lesions. The procedure entails harvesting a costal osteochondral fragment with hyaline cartilage, usually from the sixth rib, for transplantation into the recipient site on the capitellum [5, 42]. Zlotolow and Bae revealed that the costal hyaline cartilage layer is much thicker than in OATS grafts and must be trimmed in order to fit flush. The costal osteochondral graft lacks the most superficial lamina splendens layer, which aids in resisting tensile and shear forces [10]. Shimada et al. highlighted a benefit of COT in the ability to harvest larger grafts. They achieved grafts up to $15 \mathrm{~mm}$, allowing greater coverage with fewer plugs [5].

Recent literature reports promising outcomes for COT. In Shimada et al., 26 patients were treated with COT. All had capitellar lesions greater than $15 \mathrm{~mm}$ in diameter. All had improved functional outcomes and achieved radiographic union by 3 months post-operatively. By 6 months post-operatively, all athletes returned to sports. At 6 12 months, $77 \%$ of patients had congruous joint surfaces on MRI. Complications included one pneumothorax, treated with chest tube placement that ultimately resolved, and reoperation in five of the 26 patients [5]. Nishinaka et al. described their technique and outcomes in 22 overhead athletes with an average followup of 27 months. All had improved functional outcomes and achieved return to sports by an average of 7.5 months (13 at the pre-injury level and nine at a lower level). All grafts reached osseous union by an average of 3.3 months. Four patients required reoperation for loose bodies or spur formation, but all four returned to their prior level of sport after reoperation [42]. COT is a valuable alternative for large $(>15 \mathrm{~mm})$ lesions involving the lateral column. It is, however, a technically demanding operation with serious potential complications.

\section{Future directions}

Kida et al. alluded to the new wave of OCD literature in a prevalence study of capitellar OCD in adolescent baseball players. Many now contemplate the potential for screening programs in adolescent overhead throwers to diagnose OCD sooner, thus keeping earlier conservative options open. With more young athletes becoming competitive sooner, the quest for earlier and more cost-effective diagnostic imaging algorithms, i.e., the roles of radiographs, ultrasound, MRI, will drive the literature. From a treatment standpoint, we predict more literature on longer-term outcomes with regard to sports participation, level and duration, as well as resulting osteoarthritis. New literature, like that of Gancarczyk and colleagues, will contribute to the development of new, less invasive OCD therapy, i.e., arthroscopic OATS. Awareness of OCD in the general public and medical community has heightened over 
the past decade and with that so too will advances in diagnosis and treatment.

\section{Conclusion}

Capitellar osteochondritis dissecans may be more prevalent among adolescent overhead athletes than previously thought. Recent literature has brought a wealth of information targeting improved outcomes, pain relief, and return to sport. Key developments include improved outcomes after OATS procedures, COT for reconstruction of large defects, and the advances in arthroscopic in situ fixation. Current literature still supports early detection with excellent outcomes for earlystage non-operative treatment. Likewise, current literature has remained consistent in predicting poor outcomes for lateral column lesions with surgical techniques that do not restore sufficient subchondral bone support. Recent literature has largely expanded the OCD diagnostic and technical treatment armamentarium for the orthopaedic surgeon such that many of these patients now achieve good to excellent outcomes.

\section{Compliance with ethical standards}

Conflict of interest Ryan W. Churchill and Julianne Munoz declare that they have no conflict of interest.

Christopher S. Ahmad reports receiving grants and research support from Arthrex, Major League Baseball, and Stryker. He has served as a consultant for Arthrex and Acumed.

Human and animal rights and informed consent This article does not contain any studies with human or animal subjects performed by any of the authors.

\section{References}

Papers of particular interest, published recently, have been highlighted as:

- O f importance

-Of major importance

1. Konig F, The classic. The classic: on loose bodies in the joint. Clin Orthop Relat Res. 2013;471(4):1107-15.

2. Edmonds EW, Polousky J. A review of knowledge in osteochondritis dissecans: 123 years of minimal evolution from Konig to the ROCK study group. Clin Orthop Relat Res. 2013;471(4):1118-26.

3. Nissen CW. Osteochondritis dissecans of the elbow. Clin Sports Med. 2014;33(2):251-65.

4. Jones KJ et al. Arthroscopic management of osteochondritis dissecans of the capitellum: mid-term results in adolescent athletes. J Pediatr Orthop. 2010;30(1):8-13.

5. Shimada $\mathrm{K}$ et al. Cylindrical costal osteochondral autograft for reconstruction of large defects of the capitellum due to osteochondritis dissecans. J Bone Joint Surg Am. 2012;94(11): 992-1002.

6. Ahmad CS, Vitale MA, ElAttrache NS. Elbow arthroscopy: capitellar osteochondritis dissecans and radiocapitellar plica. Instr Course Lect. 2011;60:181-90.

7. Baker 3rd CL, Romeo AA, Baker Jr CL. Osteochondritis dissecans of the capitellum. Am J Sports Med. 2010;38(9):1917-28.

8. Ahmad CSENS. Treatment of capitellar osteochondritis dissecans. Techniques in Shoulder and Elbow Surgery. 2006;7(4):169-74.

9. Tis JE et al. Short-term results of arthroscopic treatment of osteochondritis dissecans in skeletally immature patients. J Pediatr Orthop. 2012;32(3):226-31.

10. Zlotolow DA, Bae DS. Osteochondral autograft transplantation in the elbow. J Hand Surg [Am]. 2014;39(2):368-72.

11. Schub DL et al. Mapping of cartilage depth in the knee and elbow for use in osteochondral autograft procedures. Am J Sports Med. 2013;41(4):903-7.

12. Claessen FM et al., Osteochondritis dissecans of the humeral capitellum: reliability of four classification systems using radiographs and computed tomography. J Shoulder Elbow Surg. 2015. Demonstrated that radiographic findings have poor to fair interobserver reliability and that MRI is needed for cases of osteochondritis dissecans of the capitellum.

13. Lewine EB et al. Early results of drilling and/or microfracture for grade IV osteochondritis dissecans of the capitellum. J Pediatr Orthop. 2015.

14. Uchida $\mathrm{S}$ et al. Arthroscopic fragment fixation using hydroxyapatite/poly-L-lactate acid thread pins for treating elbow osteochondritis dissecans. Am J Sports Med. 2015;43(5):105765. Demonstrated good results with return to play and pain relief with fragment fixation via an arthroscopic means avoiding an open arthrotomy of the elbow for fixation.

15.• Maruyama M et al. Outcomes of an open autologous osteochondral plug graft for capitellar osteochondritis dissecans: time to return to sports. Am J Sports Med. 2014;42(9):2122-7. Demonstrated return to sport in a decreased time period from previous studies of patients undergoing OATS. Patients who return to sport returned at prior or improved level.

16. $\bullet$ Kida Y et al. Prevalence and clinical characteristics of osteochondritis dissecans of the humeral capitellum among adolescent baseball players. Am J Sports Med. 2014;42(8):1963-71. Found increased prevalence of $O C D$ lesions amongst pitchers than previously reported. Found patients who had prior elbow pain and continued to pitch presented with more advanced lesions. Effectively used ultrasound as a screening tool.

17. Mihata $\mathrm{T}$ et al. Biomechanical characteristics of osteochondral defects of the humeral capitellum. Am J Sports Med. 2013;41(8): 1909-14.

18. Miyake $\mathrm{J}$ et al. In-vivo biomechanical analysis of osteochondritis dissecans of the humeral trochlea: a case report. J Pediatr Orthop B. 2013;22(4):392-6.

19. Greiwe RM, Saifi C, Ahmad CS. Pediatric sports elbow injuries. Clin Sports Med. 2010;29(4):677-703.

20. Lyons ML et al. Osteochondral autograft plug transfer for treatment of osteochondritis dissecans of the capitellum in adolescent athletes. J Shoulder Elbow Surg. 2015;24(7):1098-105. Showed good to excellent results in adolescent athletes with OATS procedures with return to play at previous level or higher in almost all patients.

21. Hennrikus WP et al. Internal fixation of unstable in situ osteochondritis dissecans lesions of the capitellum. J Pediatr Orthop. 2015;35(5):467-73.

22. Kosaka M et al. Outcomes and failure factors in surgical treatment for osteochondritis dissecans of the capitellum. J Pediatr Orthop. 2013;33(7):719-24. 
23. Iwasaki $\mathrm{N}$ et al. A retrospective evaluation of magnetic resonance imaging effectiveness on capitellar osteochondritis dissecans among overhead athletes. Am J Sports Med. 2012;40(3):624-30.

24. Dexel J et al. Comparative study of elbow disorders in young highperformance gymnasts. Int J Sports Med. 2014;35(11):960-5.

25. Zellner B, May MM. Elbow injuries in the young athlete-an orthopedic perspective. Pediatr Radiol. 2013;43 Suppl 1:S129-34.

26. Schenck Jr RC et al. A biomechanical analysis of articular cartilage of the human elbow and a potential relationship to osteochondritis dissecans. Clin Orthop Relat Res. 1994;299:305-12.

27. Haraldsson S. On osteochondrosis deformas juvenilis capituli humeri including investigation of intra-osseous vasculature in distal humerus. Acta Orthop Scand Suppl. 1959;38:1-232.

28. Kijowski R, De Smet AA. Radiography of the elbow for evaluation of patients with osteochondritis dissecans of the capitellum. Skeletal Radiol. 2005;34(5):266-71.

29. Zbojniewicz AM, Laor T. Imaging of osteochondritis dissecans. Clin Sports Med. 2014;33(2):221-50.

30. De Smet AA et al. Osteochondritis dissecans of the knee: value of MR imaging in determining lesion stability and the presence of articular cartilage defects. AJR Am J Roentgenol. 1990;155(3): 549-53.

31. Kijowski R, De Smet AA. MRI findings of osteochondritis dissecans of the capitellum with surgical correlation. AJR Am J Roentgenol. 2005;185(6):1453-9.

32. Jans LB et al. MR imaging findings and MR criteria for instability in osteochondritis dissecans of the elbow in children. Eur J Radiol. 2012;81(6):1306-10.

33. Satake $\mathrm{H}$ et al. Preoperative imaging criteria for unstable osteochondritis dissecans of the capitellum. Clin Orthop Relat Res. 2013;471(4):1137-43.

34. Harada $\mathrm{M}$ et al. Using sonography for the early detection of elbow injuries among young baseball players. AJR Am J Roentgenol. 2006;187(6):1436-41.

35. Jacobson JA. Ultrasound in sports medicine. Radiol Clin North Am. 2002;40(2):363-86.

36. Nofsinger C, Konin JG. Diagnostic ultrasound in sports medicine: current concepts and advances. Sports Med Arthrosc. 2009;17(1): 25-30.

37. Takahara $\mathrm{M}$ et al. Classification, treatment, and outcome of osteochondritis dissecans of the humeral capitellum. J Bone Joint Surg Am. 2007;89(6):1205-14.

38. Shi LL et al. Contained versus uncontained lesions in juvenile elbow osteochondritis dissecans. J Pediatr Orthop. 2012;32(3):2215 .
39. Wulf CA et al. Magnetic resonance imaging after arthroscopic microfracture of capitellar osteochondritis dissecans. Am J Sports Med. 2012;40(11):2549-56.

40. Matsuura T et al. Conservative treatment for osteochondrosis of the humeral capitellum. Am J Sports Med. 2008;36(5):868-72.

41. Mihara $\mathrm{K}$ et al. Nonoperative treatment for osteochondritis dissecans of the capitellum. Am J Sports Med. 2009;37(2):298 304.

42. Nishinaka $\mathrm{N}$ et al. Costal osteochondral autograft for reconstruction of advanced-stage osteochondritis dissecans of the capitellum. J Shoulder Elbow Surg. 2014;23(12):1888-97.

43. Baumgarten TE, Andrews JR, Satterwhite YE. The arthroscopic classification and treatment of osteochondritis dissecans of the capitellum. Am J Sports Med. 1998;26(4):520-3.

44. Brownlow HC, O'Connor-Read LM, Perko M. Arthroscopic treatment of osteochondritis dissecans of the capitellum. Knee Surg Sports Traumatol Arthrosc. 2006;14(2):198-202.

45. Byrd JW, Jones KS. Arthroscopic surgery for isolated capitellar osteochondritis dissecans in adolescent baseball players: minimum three-year follow-up. Am J Sports Med. 2002;30(4):474-8.

46. Rahusen FT, Brinkman JM, Eygendaal D. Results of arthroscopic debridement for osteochondritis dissecans of the elbow. Br J Sports Med. 2006;40(12):966-9.

47. Ruch DS, Cory JW, Poehling GG. The arthroscopic management of osteochondritis dissecans of the adolescent elbow. Arthroscopy. 1998;14(8):797-803.

48. Bojanic I, Smoljanovic T, Dokuzovic S. Osteochondritis dissecans of the elbow: excellent results in teenage athletes treated by arthroscopic debridement and microfracture. Croat Med J. 2012;53(1): 40-7.

49. Nishimura A et al. Functional recovery of the donor knee after autologous osteochondral transplantation for capitellar osteochondritis dissecans. Am J Sports Med. 2011;39(4):838-42.

50. Iwasaki $\mathrm{N}$ et al. Donor site evaluation after autologous osteochondral mosaicplasty for cartilaginous lesions of the elbow joint. Am J Sports Med. 2007;35(12):2096-100.

51. Gancarczyk SM et al., Arthroscopic articular reconstruction of capitellar osteochondral defects. Am J Sports Med, 2015. Showed via a cadaveric model that the vast majority of OCD lesions of the capitellum can be approached in an acceptable amount of perpendicularity via arthroscopic means. This may provide a future for lower morbidity surgery through arthroscopic methods for performing an OATS procedure. 